\title{
Stochastic Processes that Generate. Polygonal and Related Random Fields
}

\author{
Vivek S. Borkar, Senior Member, IEEE, and Sanjoy K. Mitter, Fellow, IEEE
}

\begin{abstract}
A reversible, ergodic, Markov process taking values in the space of polygonally segmented images is constructed. The stationary distribution of this process can be made to correspond to a Gibbs-type distribution for polygonal random fields as introduced by Arak and Surgailis and a few variants thereof, such as those arising in Bayesian analysis of random fields. Extensions to generalized polygonal random fields are presented where the segmentation boundaries are not necessarily straight line segments.
\end{abstract}

Index Terms-Polygonal random fields, generalized polygonal random fields, reversible Markov process, interacting particle system, Monte Carlo simulation of random fields.

\section{INTRODUCTION}

I N A remarkable series of papers, Arak and Surgailis [1]-[3] studied a class of Markov random fields called polygonal random fields (PRF's) whose realizations can be construed as polygonally segmented images. An important aspect of this work is the specification of an interacting particle system on the line with certain birth, death, branching, and annihilation mechanisms, whose trace in the space-time domain gives a realization of the PRF. Since PRF's provide a convenient model for polygonally segmented images, it is important to be able to construct a reversible Markov process taking values in the space of possible PRF realizations such that its sample at any given time gives a PRF realization. with the desired statistics. This is needed, e.g., for Bayesian reconstruction of a polygonally segmented image by Monte Carlo methods. Motivated by this, Clifford [4], Clifford and Middleton [5], and Judish [6] proposed schemes for constructing such processes. Their algorithms proceed by modifying at each step the present realization of the $\mathrm{PRF}$ on a randomly chosen rectangular subdomain, so as to achieve the desired Gibbs distribution. These algorithms, however, are strewn with many analytic and computational difficulties, discussed at length in [6]. Our aim here is to provide a simpler alternative scheme which explicitly uses the Arak-Surgailis particle dynamics. This scheme also leads to an important generalization to Markov random fields exhibiting polygonal-like segmentations, but with curved (as

Manuscript received March 15, 1994; revised April 12, 1995. This work was supported by the US Army Research Office under Grant ARO DAAL0392-G-0115 (Center for Intelligent Control Systems).

V. S. Borkar is with the Department of Electrical Engineering, Indian Institute of Science, Bangalore-560 012, India.

S. K. Mitter is with the Department of Electrical Engineering and Computer Science and Laboratory for Information and Decision Systems, Massachusetts Institute of Technology, Cambridge, MA 02139 USA.

Publisher Item Identifier S 0018-9448(96)01020-6. opposed to straight-line) boundaries. We call these generalized polygonal random fields (GPRF's).

The paper is organized as follows: The next section describes the notation and the Arak-Surgailis framework. The Arak-Surgailis particle system is described next in Section III. Section IV describes our.construction of a process taking values in PRF realizations. Section V describes the extension to GPRF's.

\section{PRELIMINARIES}

Let $T \subset \mathcal{R}^{2}$ be a bounded, open, convex domain. Parameterize the straight lines in $\mathcal{R}^{2}$ by $(p, \alpha) \in \mathcal{R} \times[0, \pi)$ where $p$ is the signed length of the perpendicular to line $\ell$ from the origin and $\alpha$ the angle it makes with the horizontal axis. Let $\mathcal{L}_{T}$ denote the set of all straight lines in $\mathcal{R}^{2}$ that intersect $T$ and $\mathcal{L}_{T, n}^{0}$ the set of $n$-tuples of distinct elements of $\mathcal{L}_{T}$. Let $J$ be a prescribed finite set of "colors." Define

$$
\Omega_{T}(\ell)_{n}=\{\omega: T \rightarrow J \mid \partial \omega \triangleq
$$

the set of points of discontinuity of $\omega$, satisfies:

$$
\partial \omega \subset \bigcup_{i=1}^{n} \ell_{i} \bigcap T \quad \text { where }(\ell)_{n} \triangleq\left\{\left[\ell_{1}, \cdots, \ell_{n}\right] \in \mathcal{L}_{T, n}^{0}\right\}
$$

To avoid any ambiguity in the definition of $\omega \in \Omega_{T}(\ell)_{n}$ on $\partial \omega$, we further impose the condition

$$
\omega(z)=\inf _{S} \limsup _{\varepsilon \downarrow 0}\left\{\omega\left(z^{\prime}\right) \mid z^{\prime} \in T \backslash S,\left\|z^{\prime}-z\right\|<\varepsilon\right\}
$$

where the infimum is over all $S \subset T$ of Lebesgue measure zero, with respect to an arbitrary but fixed ordering of $J$. Let

$$
\Omega_{T}=\bigcup_{n=0}^{\infty} \bigcup_{(\ell)_{n} \in \mathcal{L}_{T, n}^{a}} \Omega_{T}(\ell)_{n}
$$

This is the space of "polygonally segmented images," topologized as follows: A local base for the topology at

$$
\omega \in \bigcup_{(\ell)_{n} \in \mathcal{L}_{T, n}^{0}} \Omega_{T}(\ell)_{n}
$$

is given by sets of the type

$$
\begin{gathered}
\left\{\omega^{\prime} \in \Omega_{T} \mid \omega^{\prime} \in \bigcup_{(\ell)_{n} \in \mathcal{L}_{T, n}^{0}} \Omega_{T}(\ell)_{n}, \partial \omega^{\prime} \in(\partial \omega)^{\varepsilon}\right. \\
\left.\omega^{\prime}=\omega \text { on }\left((\partial \omega)^{\varepsilon}\right)^{c}\right\}
\end{gathered}
$$




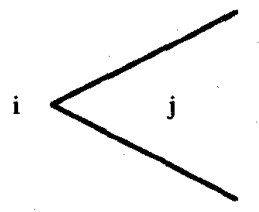

(a)

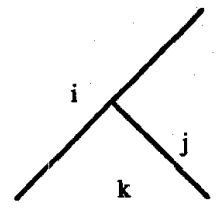

(b)

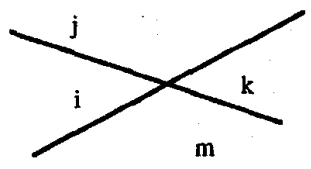

(c)

Fig. 1. Nodes of different kind. (a) Corner. (b) $\mathrm{T}$-junction. (c) $\mathrm{X}$-junction.

where we use the notation

$$
A^{\varepsilon} \triangleq\left\{x \in T \mid \inf _{y \in A}\|x-y\|<\varepsilon\right\}
$$

for $A \subset T, \varepsilon>0$. We endow $\Omega_{T}$ with the corresponding Borel $\sigma$-field $\mathcal{B}_{T}$.

Let $\mu=\mu(d \ell)$ be a finite, nonatomic, nonnegative measure on $\mathcal{L}_{T}$. Define the set of "admissible potentials"

$$
\begin{aligned}
\Phi_{T, \mu}=\{F: & \Omega_{T} \rightarrow \mathcal{R} \cup\{\infty\} \mid Z_{T, F, \mu} \\
\triangleq & \sum_{n=0}^{\infty} \int_{\mathcal{L}_{T, n}^{0}} \frac{1}{n !} \mu\left(d \ell_{1}\right) \cdots \mu\left(d \ell_{n}\right) \\
& \left.\times \sum_{\omega \in \Omega_{T}(\ell)_{n}} e^{-F(\omega)}<\infty\right\} .
\end{aligned}
$$

A polygonal random field on $T$ corresponding to measure $\mu$ and potential $F \in \Phi_{T, \mu}$ is the probability measure $P_{T}=$ $P_{T, F, \mu}$ on $\left(\Omega_{T}, \mathcal{B}_{T}\right)$ given by

$$
\begin{aligned}
P_{T}(A)= & \sum_{n=0}^{\infty} \int_{\mathcal{L}_{T, n}^{0}} \frac{1}{n !} \mu\left(d \ell_{1}\right) \cdots \mu\left(d \ell_{n}\right) \\
& \times \sum_{\omega \in \Omega_{T}(\ell)_{n} \cap A} e^{-F(\omega)} / Z_{T}, \quad A \in \mathcal{B}_{T} .
\end{aligned}
$$

Remark 2.1: In [2], Arak and Surgailis give a somewhat more general definition allowing for $\mu$ that are not nonatomic. But the specific $\mu$ that they use later on in [2] is nonatomic. We shall be using the same choice of $\mu$.

Recall our parametrization of $\ell \in \mathcal{L}_{T}$. A random sequence of lines $\ell_{j}, j \geq 1, \ell_{j} \approx\left(p_{j}, \alpha_{j}\right)$, is said to be a Poisson line process with intensity $\mu(d \ell)$ if $\left(p_{j}, \alpha_{j}\right), j \geq 1$, is a Poisson point process on $\mathcal{R} \times[0, \pi)$ with intensity $\mu(d p, d \alpha)$. It is stationary if and only if $\mu(d \ell)=\mu(d p, d \alpha)$ is of the form $d p \nu(d \alpha)$ for a bounded nonnegative measure $\nu$ on $[0, \pi)$. Motivated by image processing applications, we shall be interested in stationary isotropic PRF's, i.e., those PRF's whose satistics is invariant under Euclidean motions and reflections. Therefore, we take (as in [2]) $\nu(d \alpha)=d \alpha$.

The next step is to choose $F(\cdot)$. Given $\omega \in \Omega_{T}$, let a "node" of $\omega$ refer to any point in $T$ that belongs to more than one distinct line segment of $\partial \omega$. Fig. 1 describes three kinds of nodes $(i, j, k, m$ stand for colors in $J)$.

Let $n_{2}(i, j)(\omega), n_{3}(i ; j, k)(\omega), n_{4}(i, j, k, m)(\omega)$ denote the number of such corners, $T$-junctions, and $X$-junctions, respectively.
Define $F(\cdot)$ by

$$
\begin{aligned}
-F(\omega)= & \frac{1}{2} \sum_{i \neq j} n_{2}(i, j)(\omega) \log b(i, j) \\
& +\frac{1}{2} \sum_{i \neq k \neq j} n_{3}(i ; j, k)(\omega) \log c(i ; j, k) \\
& +\sum_{i, j, k, m} c^{\prime}(i, j, k, m) n_{4}(i, j, k, m)(\omega) \log d(i, j, k, m) \\
& +\frac{1}{2} \sum_{i, j} \sum_{[\ell] \in \partial \omega(i, j)}(\log a(i, j)-\lambda e(i, j) L([\ell])) \\
& -\sum_{i} f(i)\left|T_{i}(\omega)\right|
\end{aligned}
$$

where "log" denotes the natural logarithm (with $\log 0 \triangleq-\infty$ ) and

i) $\lambda=\int_{0}^{\pi}|\sin \beta| d \beta$,

ii) $c^{\prime}(i, j, k, m)=\frac{1}{8}$ if $i, j, k, m$ are distinct, $=\frac{1}{4}$ if $i \neq k, j=m$ or $i=k, j \neq m,=\frac{1}{2}$ if $i=k, j=m$,

iii) $T_{i}(\omega)=\{z \in T \mid w(z)=i\} \backslash \partial \omega,\left|T_{i}(\omega)\right|$ its area,

iv) $a(i, j), b(i, j), c(i, j, k), c^{\prime}(i, j, k, m), d(i, j, k, m)$, $e(i, j)$ are nonnegative weights satisfying $[2$, conditions (5.5)-(5.8), (5.12)-(5.18)], recalled in the Appendix. These conditions involve a symmetric transition matrix $\left[\left[p_{i j}\right]\right]_{i, j \in J}, p_{i j}=p_{j i}$, on $J$.

v) $[\ell]$ denotes a line segment belonging to line $\ell$ and $\partial \omega(i, j)$ the set of all $(i, j)$-segments, i.e., line segments in $\partial \omega$ that separate colors $i$ and $j$ in $\omega . L(\cdots)$ denotes "the length of ...."

vi) For $\omega \in \Omega_{T}(\ell)_{n}, \ell=\left[\ell_{1}, \cdots, \ell_{n}\right]$, the set $\partial \omega \bigcap \ell_{i}$, when nonempty, is a single line segment for each $i$.

We set $F(\omega)=-\infty$ if $\partial \omega$ contains a node of any type other than those described in Fig. 1. This is not a serious restriction because other kinds of nodes (such as more than two line segments meeting or crossing at a point) are structurally unstable, i.e., become qualitatively different under arbitrarily small perturbations.

For $S \subset T$ open, let $\pi_{S}(\omega) \in \Omega_{S}$ for $\omega \in \Omega_{T}$ denote the restriction of $\omega$ to $S$ and let $\mathcal{B}_{S}$ denote the sub- $\sigma$-field of $\mathcal{B}$ generated by the map $\pi_{S}: \Omega_{T} \rightarrow \Omega_{S}$. A measurable map $X: \Omega_{T} \rightarrow R \cup\{\infty\}$ is said to be additive if, whenever $T=S \bigcup V, S, V$ open, $X=X_{S}+X_{V}$ for some $X_{S}$, $X_{V}: \Omega_{T} \rightarrow R \cup\{\infty\}$ which are, respectively, $\mathcal{B}_{S}, \mathcal{B}_{V^{-}}$ measurable. (This decomposition need not be unique.) With this definition, the potential $F(\cdot)$ above is seen to be additive.

The polygonal random field $P_{T}$ is said to be Markov if for $S, V$ as above and any $A \subset \mathcal{B}_{S}, P_{T}\left(A / \mathcal{B}_{V}\right)=P_{T}\left(A / \mathcal{B}_{V \cap S}\right)$. Let $\mathcal{G}_{0}$ denote the set of bounded convex open sets in $\mathcal{R}^{2}$. 
Theorem 2.1 [2, Theorem 5.1]: For the above choice of $\mu$ and $F$ as in (2.1), the probability measures $P_{T}, T \in \mathcal{G}_{0}$, define a consistent family of isotropic Markov PRF's.

The next result characterizes the conditional distribution under $P_{T}$.

Theorem 2.2 [2, Lemma 8.3]: For $A \in \mathcal{B}, U \subset T$ open

$$
P_{T}\left(A / \mathcal{B}_{U}\right)(\omega)=Z_{T \backslash U}\left(A / \pi_{U}(\omega)\right) / Z_{T \backslash U}(\pi(\omega)) .
$$

Here for $\xi \in \Omega_{U}$

$$
Z_{T \backslash U}(A / \xi)=\sum_{m=0}^{\infty} \int_{\mathcal{L}_{T \backslash U, m}^{0}} \frac{1}{m !} d^{m} \mu(\ell) \Sigma^{\xi} I_{A}(\omega) e^{-F(\omega)}
$$

where the sum $\Sigma^{\xi}$ is over all $\omega \in \Omega_{T}$ satisfying $\pi_{U}(\omega)=\xi$ and $\omega \in \Omega_{T}\left((\ell)_{m} \cup \tilde{L}(\xi)\right), \tilde{L}(\xi)$ being the set of lines that constitute $\xi$.

$$
Z_{T \backslash U}\left(\pi_{U}(\omega)\right)=Z_{T \backslash U}\left(\Omega_{T} / \pi_{U}(\omega)\right)
$$

is the normalizing factor.

The proof of [2, Theorem 2.1] uses the realization of these PRF's via an interacting particle system with prescribed dynamics. We describe this in the next section. In conclusion, we mention that the definition of isotropy in [2] does not include reflection symmetry, but this can be easily incorporated without altering the proof of Theorem 2.1.

\section{DYNAMICS OF THE PARTICle SYSTEM}

We start with further notation and definitions from [2]. We consider a $T$ which is a bounded convex polygon. Without any loss of generality, suppose that

$$
T \subset[0,1]^{2} \triangleq\{(t, y) \mid 0 \leq t, y \leq 1\}
$$

with no side parallel to the $y$-axis. This can always be achieved by redefining the axes and scaling--see, e.g., Fig. 2 . In particular, $\bar{T}$ has one point each on the lines $(0, y)$ and $(1, y)$. Let $\partial T=\partial T^{+} \cup \partial T^{-}$where $\partial T^{ \pm}=\left\{\left(t, y_{t}^{ \pm}\right), 0 \leq\right.$ $t \leq 1\}$ are the upper and lower parts of $\partial T=$ the boundary of $T$, so that $T=\left\{(t, y) \mid 0<t<1, y_{t}^{-}<y<y_{t}^{+}\right\}$. We interpret the $t$-axis as the time axis. By a particle we mean a quadruple $z=(y, v, i, j)$ where $y \in[0,1]$ is its position, $v \in \mathcal{R}$ its velocity, and $i, j \in J, i \neq j$, are the "environments" above and below the particle, respectively. Call such a particle an $(i, j)$-particle. A system of particles is a finite collection

$$
z=\left(z_{1}, \cdots, z_{n}\right), \quad z_{r}=\left(y_{r}, v_{r}, k_{r}^{+}, k_{r}^{-}\right)
$$

of particles such that $\left(y_{r}, v_{r}\right) \neq\left(y_{s}, v_{s}\right)$ for $s \neq r$. The system is said to be ordered if for $1 \leq r<n$, either $y_{r}<y_{r+1}$ or $y_{r}=y_{r+1}, v_{r}<v_{r+1}$. Any system of $n$ particles can be ordered by a permutation of its indices. An ordered system is said to be consistent if $k_{r}^{+}=k_{r+1}^{-}, 1 \leq r<n$. Let $X^{(n)}$, $n \geq 1$, denote the set of ordered consistent systems of $n$ particles and for $t \in[0,1], X_{t}^{(n)} \subset X^{(n)}$ its subset consisting of these systems for which for $1 \leq r \leq n$, either

$$
y_{t}^{-}<y_{r}<y_{t}^{+}
$$

or

or

$$
y_{r}=y_{t}^{-}, \quad v_{r}>v_{t}^{-}
$$

$$
y_{r}=y_{t}^{+}, \quad v_{r}<v_{t}^{+}
$$

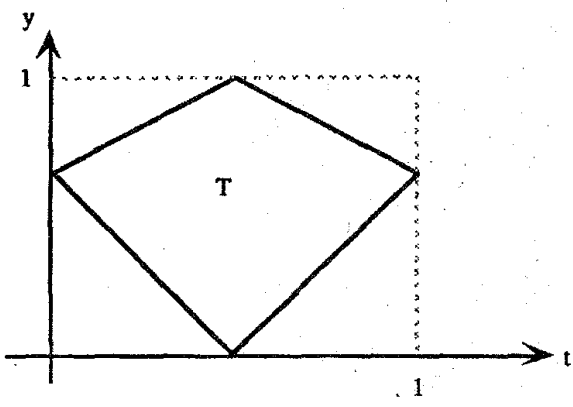

Fig. 2. Redefinition of axes.

holds, where

$$
v_{t}^{ \pm}=\lim _{s \rrbracket t} \frac{d y_{s}^{ \pm}}{d s}
$$

is the tangent to $\partial T^{ \pm}$at $t$. Set $X^{(0)}=X_{t}^{(0)}=J$ and

$$
\begin{gathered}
X=\bigcup_{n=0}^{\infty} X^{(n)} \\
X_{t}=\bigcup_{n=0}^{\infty} X_{t}^{(n)} .
\end{gathered}
$$

For any $x=\left[z_{1}, \cdots, z_{n}\right] \in X_{t}^{(n)}, n \geq 1$, define its environment as the right-continuous function $w(\cdot, x):\left(y_{t}^{-}, y_{t}^{+}\right) \rightarrow J$ such that

$$
w(y, x)= \begin{cases}k_{r}^{+}=k_{r+1}^{-}, & \text {if } y \in\left(y_{r}, y_{r+1}\right), 1 \leq r \leq n \\ k_{1}^{-}, & \text {if } y \in\left(y_{t}^{-}, y_{1}\right) \\ k_{n}^{+}, & \text {if } y \in\left(y_{n}, y_{t}^{+}\right)\end{cases}
$$

for $y \neq y_{1}, \cdots, y_{n}$. For $x=k \in X_{t}^{(0)}$, set $w(y, x)=k$, $y \in\left(y_{t}^{-}, y_{t}^{+}\right)$. The evolution of the particle system as a Markov process taking values in $X_{t}$ at time $t$ is described by i)-x) below:

i) The initial distribution of $x(t)$ at $t=0$ is concentrated on $X_{0}^{(0)}=J$ with $P(x(0)=j)=1 /|J|$. Let $x(t)=$ $x \in X_{t}^{(n)}$ be the value of $x(t)$ at time $t \in[0,1)$. In a small time interval $(t, t+\Delta t) \subset[0,1]$, the following changes can occur.

ii) With probability $p_{i j} q\left(v_{t}^{+}, d u\right) \Delta t+o(\Delta t)$, a new particle $(y, v, i, j)$ is born at $\partial T^{+}$with $j=k_{n}^{+}, v \in d u$, $v<v_{t}^{+}$, where

$$
q(v, d u)=|u-v| d u d t /\left(1+u^{2}\right)^{3 \backslash 2} .
$$

iii) With probability $p_{i j} q\left(v_{t}^{-}, d u\right) \Delta t+o(\Delta t)$ a new particle $(y, v, i, j)$ is born at $\partial T^{-}$with $i=k_{1}^{-}, v \in d u, v>v_{t}^{-}$.

iv) With probability $p_{i j}^{2} b(i, j)\left|u^{\prime}-u^{\prime \prime}\right| V\left(d u^{\prime}\right) V\left(d u^{\prime \prime}\right) d y \Delta t$ $+o(\Delta t)$ two new particles $\left(y, v^{\prime}, i, j\right)$ and $\left(y, v^{\prime \prime}, i, j\right)$ are born with $y \in d y \subset\left(y_{t}^{-}, y_{t}^{+}\right), i=w(y, x)$, $v^{\prime} \in d u^{\prime}, v^{\prime \prime} \in d u^{\prime \prime}, v^{\prime}>v^{\prime \prime}$

$$
V(d u)=|\{\alpha \in(0, \pi) \mid \cot (\alpha) \in d u\}| / \sqrt{1+u^{2}} .
$$

v) With probability $p_{i j} b(i, j) q(v, d u) \Delta t+o(\Delta t)$, one of the particles ${ }^{*} z_{r}, 1 \leq r \leq n, z_{r}=(y, v, i, j)$, turns into the particle $\left(y, v^{\prime}, i, j\right)$ with $v^{\prime} \in d u$. 


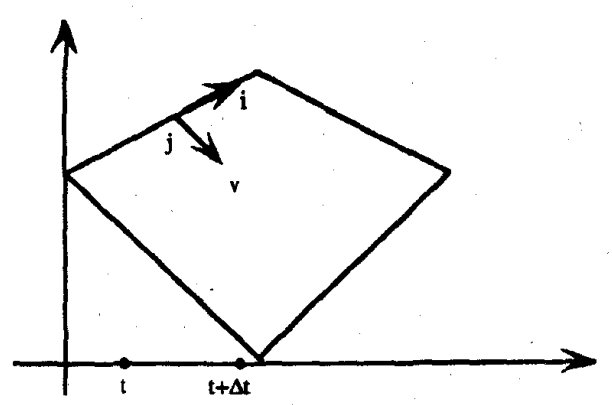

Fig. 3. Birth of particles.

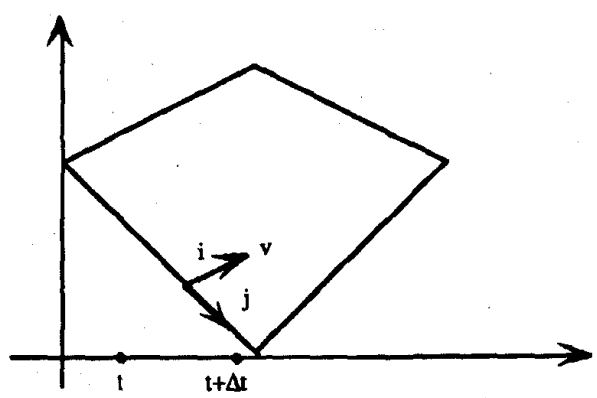

Fig. 4. Birth of particles.

vi). With probability $p_{i j} p_{k j} c q(v, d u) \Delta t+o(\Delta t)$, one of the particles $z_{r}, 1 \leq r \leq n, z_{r}=(y, v, i, j)$, turns into either two new particles $(y, v, i, k),\left(y, v^{\prime}, k, j\right)$ with $v^{\prime} \in d u, v^{\prime}<v, c=c(i ; j, k)$ or into two new particles $(y, v, k, j),\left(y, v^{\prime}, i, k\right)$ with $v^{\prime} \in d u, v^{\prime}>v$, $c=c(j ; i, k)$.

vii) With probability

$$
\begin{aligned}
1 & -\{q(v,\{u>v\})+q(v,\{u<v\}) \\
& +\int_{y_{t}^{-}}^{y_{t}^{+}} f(w(y, x)) d y \\
& +\sum_{r=1}^{n}\left(q\left(v_{r},\left\{u<v_{r}\right\}\right) e\left(k_{r}^{+}, k_{r}^{-}\right)\right. \\
& \left.\left.+q\left(v_{r},\left\{u>v_{r}\right\}\right) e\left(k_{r}^{-}, k_{r}^{+}\right)\right)\right\} \Delta t+o(\Delta t)
\end{aligned}
$$

none of the changes in ii)-vi) occur and

$$
x(t+\Delta t)=\left[z_{1}^{\prime}, \cdots, z_{n}^{\prime}\right] \in X_{t+\Delta t}^{(n)}
$$

where

$$
z_{r}^{\prime}=\left(x_{r}+v_{r} \Delta t, v_{r}, k_{r}^{+}, k_{r}^{-}\right), 1 \leq r \leq n
$$

In ii)-vii) above, $\Delta t$ is assumed to be so small that the particles do not hit $\partial T$ or collide. If $z_{r}=(y, v, i, j)$ and $z_{r+1}=(y, u, j, k)$ collide at $(t, y)$ with $u>v$, then:

viii) If $i=k$, with probability $b(i, j)$ both die, or, with probability $d(i, j, i, m) p_{i m}^{2}$, they turn into two new particles $(y, v, m, i),(y, u, i, m)$ for $i \neq m \in J$.

ix) If $i \neq k$, then:

ixa) With probability $c(k ; i, j) p_{i k}$, they merge into a single particle $(y, u, i, k)$,

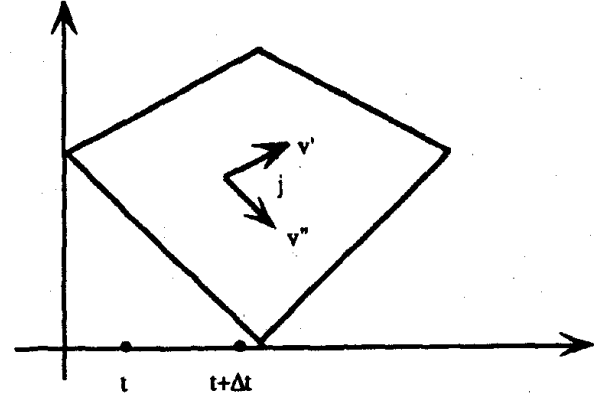

Fig. 5. Birth of particles.

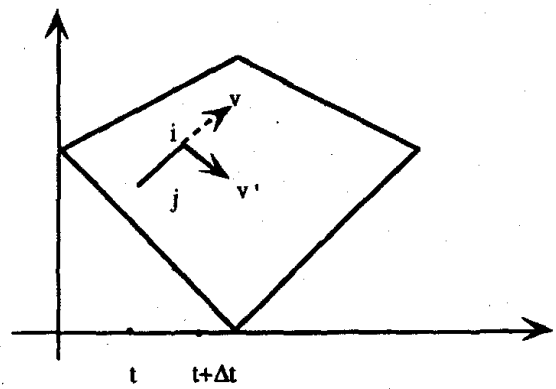

Fig. 6. Sequence of events after birth of particles.

ixb) With probability $c(i ; j, k) p_{i k}$, they merge into a single particle $(y, v, i, k)$

ixc) With probability $d(i, j, k, m) p_{i m} p_{k m}$ they turn into two particles $(y, u, i, m),(y, v, m, k), m \neq$ $i, k, m \in J$.

x) If one of the particles (say, $z_{n}$ ) reaches $\partial T$ at time $t$, it dies and the process $x(\cdot)$ jumps from $x(t-)=x$ to $x(t)=x^{\prime}=\left[z_{1}, \cdots, z_{n-1}\right] \in X_{t}^{(n-1)}$.

Figs. 3-9 illustrate the events ii)-vi), viii), and ix), respectively.

There exists a Markov process $x(\cdot)$ evolving as per $\mathrm{i}$ )- $\mathrm{x}$ ) above and to each trajectory $x(\cdot)$ thereof there corresponds a unique polygonally segmented image given by

$$
w(t, y)=w_{x(\cdot)}(t, y)=w(y, x(t)), \quad(t, y) \in T \backslash \partial \omega .
$$

Let $Q_{T}$ be the probability measure induced by this random element of $\Omega_{T}$ on $\left(\Omega_{T}, \mathcal{B}_{T}\right)$.

Theorem 3.1 [2, Lemma 6.1]: $Q_{T}=P_{T, F, \mu}$.

\section{Process of Polygonal Random FIELdS}

Our aim is to construct an $\Omega_{T}$-valued reversible ergodic process such that at each time $t$ it yields a PRF with a prescribed additive potential $H$ satisfying

$$
\{\omega \mid F(\omega)=\infty\} \subset\{\omega \mid H(\omega)=\infty\} .
$$

We shall consider the specific case of $T=$ a rectangle. The case $H=F$ is the simplest and we consider it first. In accordance with Fig. 2, draw $T$ as shown in Fig. 10. We have marked its corners as $a, b, c, d$, while $e, f$ are midpoints of $a d, b c$, respectively. The unit vector $\alpha$ is directed along the 


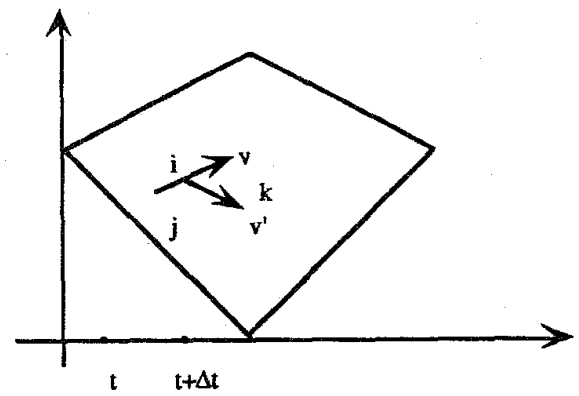

(a)

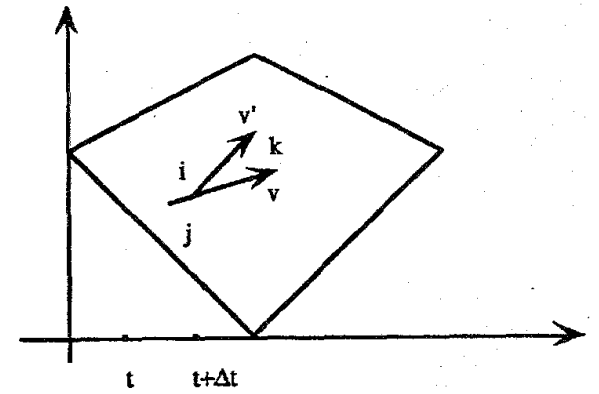

(b)

Fig. 7. Sequence of events after birth of particles. (a) $v<v^{\prime}$. (b) $v^{\prime}>v$.

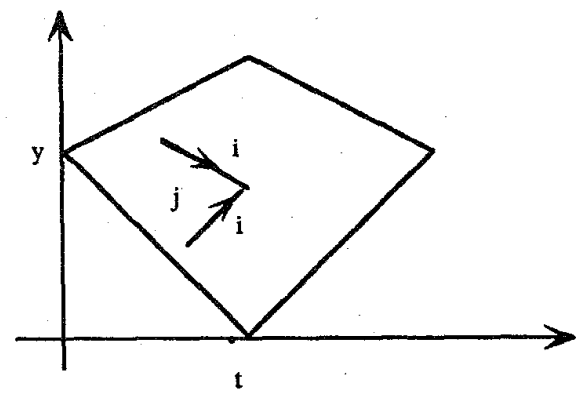

Fig. 8. Diagrammatic description of events.

perpendicular from the origin to $a b$ and $\theta$ is the angle it makes with the positive $t$-axis. Let $\hat{T}$ (respectively, $\tilde{T}$ ) denote the one-sided (respectively, two-sided) infinite "cylinder" obtained from $T$ by dropping $c d$ (respectively, $a b$ and $c d$ ) and extending $a d, b c$ indefinitely. (See Fig. 11.) Construct a system of particles evolving as in Section II on $\hat{T}$, except that it is now allowed to go on indefinitely, i.e., $x(t)$ is now defined for $t \in[0, \infty)$. Define a rectangle-valued process $T(t), t \in \mathcal{R}$ by $T(0)=T, T(t)=T+\alpha t$. Define an $\Omega_{T}$-valued process $\xi_{t}, t \geq 0$, by

$$
\xi_{t}(s, y)=\omega(s+t \cos \theta, y+t \sin \theta), \quad(s, y) \in \mathcal{T}, t \geq 0 .
$$

Call an $\Omega_{T}$-valued process $\left\{\Gamma_{t}, t \geq 0\right\} R$-reversible if for any $t_{0}>0,\left\{\Gamma_{t}, t \in\left[0, t_{0}\right]\right\}$ and $\left\{R\left(\Gamma_{t_{0}-t}\right), t \in\left[0, t_{0}\right]\right\}$ agree in law, where $R: \Omega_{T} \rightarrow \Omega_{T}$ is the map that maps $\omega \in \Omega_{T}$ to its reflection across the line $e f$ in Fig. 2 .

Lemma 4.1: $\xi_{t}, t \geq 0$, is a stationary $R$-reversible Markov process.

This is immediate from the isotropy and Markov property of $P_{T}$. In particular, $R$-reversibility allows us to symmetrically define $\xi_{t}$ for $t \leq 0$. Thus we consider $x(t)$ and $\xi_{t}$ as being defined for $t \in \mathcal{R}$.

Theorem 4.1: $\xi_{t}, t \in \mathcal{R}$ is ergodic.

Proof: Let $t>0$ be such that $\overline{T(t)} \cap \overline{T(0)}=\phi$. Let $C=$ the convex, hull of $T(0)$ and $T(t)$ and let $\omega^{\prime}, \omega^{\prime \prime} \in \Omega_{T}$. For any $\bar{\omega} \in \Omega_{T(t)}$, we can always introduce an appropriate number of births, deaths, branching, etc., in $C \backslash(\overline{T(0) \cup T(t)})$ to construct a valid trajectory of $x(\cdot)$ that restricts to $\omega^{\prime}$ (respectively, $\left.\omega^{\prime \prime}\right)$ on $\Omega_{T(0)}$ and to $\bar{\omega}$ on $\Omega_{T(t)}$. Let $\varphi_{t}: \Omega_{T(0)} \rightarrow \Omega_{T(t)}$

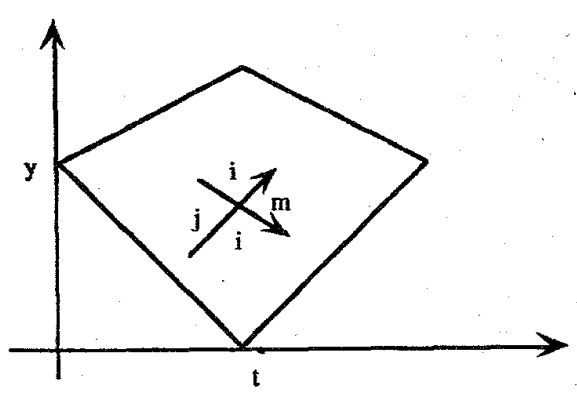

denote the map

$$
(s, y) \rightarrow(s+t \cos \theta, s+t \sin \theta)
$$

and let

$$
A^{\prime}=\left\{\omega \in \Omega_{C}|\omega|_{\Omega_{T(t)}} \in \varphi_{t}(A)\right\}, \quad A \in B
$$

Then

$$
\begin{aligned}
P\left(\xi_{t} \in A / \xi_{0}\right. & \left.=\omega^{\prime}\right) \\
& =Z_{C \backslash T(0)}\left(A^{\prime} / \pi_{T(0)}\left(\omega^{\prime}\right)\right) / Z_{C \backslash T(0)}\left(\pi_{T(0)}\left(\omega^{\prime}\right)\right)
\end{aligned}
$$

in the notation of Theorem 2.2, with a similar expression for $P\left(\xi_{i} \in A / \xi_{0}=\omega^{\prime \prime}\right)$. From the explicit expressions for the right hand side derived from Theorem 2.2 , it follows that the probability measures $P\left(\xi_{t} \in d \omega / \xi_{0}=\omega^{\prime}\right), P\left(\xi_{t} \in d \omega / \xi_{0}=\right.$ $\left.\omega^{\prime \prime}\right)$ are mutually absolutely continuous. Thus if $\left\{\xi_{t}\right\}$ has two invariant probability measures, they must be mutually absolutely continuous. Since distinct ergodic measures must be mutually singular, the claim follows.

The next two lemmas establish some additional properties of $\left\{\xi_{t}\right\}$. Let $T_{1}, T_{2}, T_{3}$ denote the open rectangles $a b f^{\prime \prime} e^{\prime \prime}$, $e^{\prime} f^{\prime} c d, e^{\prime \prime} f^{\prime \prime} c d$, respectively, in Fig. 10. For $S, U \subset T$ open, say that $w_{1} \in \Omega_{S}, \omega_{2} \in \Omega_{U}$ are compatible if they are the restrictions to $S, U$ respectively of some $\omega \in \Omega_{T}$. For $\omega_{1} \in \Omega_{T}$, the trace of $\omega_{1}$ on $e^{\prime \prime} f^{\prime \prime}$, denoted by $\operatorname{tr}\left(\omega_{1}\right)$, is an alternating sequence of colors, points of $e^{\prime \prime} f^{\prime \prime}$, and scalars, say $i_{1}, x_{1}, v_{1}, i_{2}, x_{2}, v_{2}, \cdots, i_{n}, x_{n}, v_{n}, i_{n+1}$, with the following interpretation: Under $\omega_{1}$, if we move from $e^{\prime \prime}$ to $f^{\prime \prime}$ along $e^{\prime \prime} f^{\prime \prime}$ looking at the immediate neighborhood in $T_{1}$, we first encounter a patch of color $i_{1}$, till at $x_{1}$ a trajectory from $\omega_{1}$ 


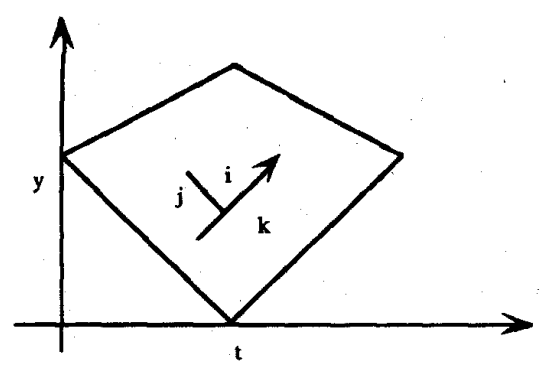

(a)

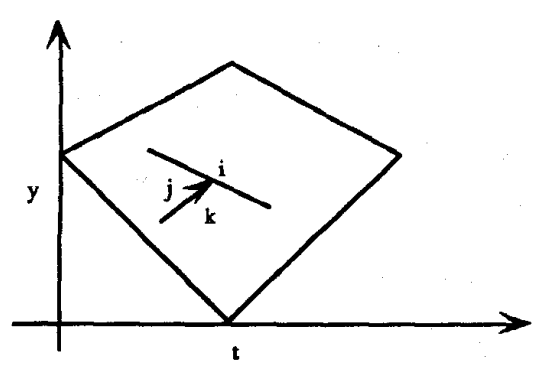

(b)

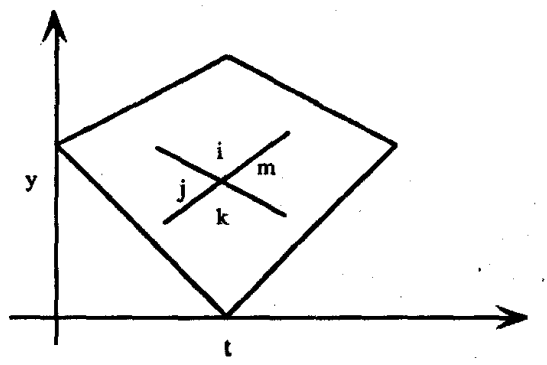

(c)

Fig. 9. Diagrammatic description of events.

hits $e^{\prime \prime} f^{\prime \prime}$ with velocity $v_{1}$. This is followed by a patch of color $i_{2}$ and so on. Clearly, $i_{k} \neq i_{k+1}, 1 \leq k \leq n$. (Situations such as $x_{1}=e^{\prime \prime}$ are also possible and can be handled analogously.) Let $\omega_{1}, \omega_{2} \in \Omega_{T_{1}}$ with

$$
\operatorname{tr}\left(\omega_{k}\right)=\left(i_{1}^{k}, x_{1}^{k}, v_{1}^{k}, \cdots, i_{m_{k}+1}^{k}\right), \quad k=1,2 .
$$

Let $d\left(\operatorname{tr}\left(\omega_{1}\right), \operatorname{tr}\left(\omega_{2}\right)\right)=\infty$ if either $m_{1} \neq m_{2}$ or $m_{1}=m_{2}$ but $i_{k}^{1} \neq i_{k}^{2}$ for some $k$, and $=\max _{i}\left(\left|x_{i}^{1}-x_{i}^{2}\right|,\left|v_{i}^{\pi}-v_{i}^{2}\right|\right)$ otherwise. It is clear that if $\omega_{n} \rightarrow \omega$ in $\Omega_{T_{1}}, \operatorname{tr}\left(\omega_{n}\right) \rightarrow \operatorname{tr}(\omega)$ w.r.t. the metric ' $d$ '.

Recall our definition of nodes. We call these interior nodes to distinguish them from boundary nodes which are points on the boundary where a particle is born or dies. For $\omega \in \Omega_{T}$, let separation of $\omega$, denoted by $\operatorname{sep}(\omega)$, be the minimum of the distances between any two nodes of either variety, between a node and any line segment in $\partial \omega \cup \partial T$ that does not contain it, the angles between any line segment in $\partial \omega$ and the $y$-axis, or the angles beween any two distinct line segments in $\partial \omega \cup \partial T$ that meet at a point. Let $N(\omega)=$ the number of distinct line segments in $\omega$.

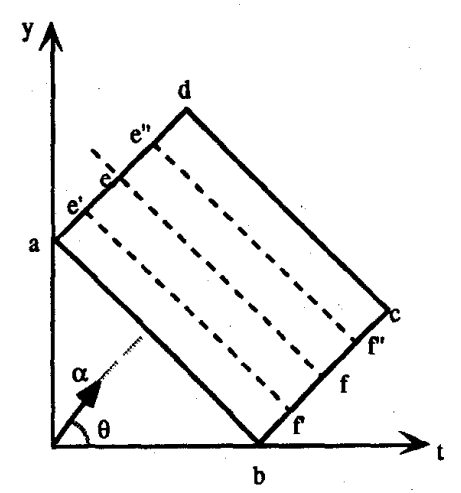

Fig. 10, Redrawing of domain $T$ in accordance with Fig. 2.

Lemma 4.2: $\left\{\xi_{t}\right\}$ is a Feller process.

Before proving this result, we first reduce it to another equivalent claim. Note that it suffices to show that for $f \in$ $C_{b}\left(\Omega_{T_{2}}\right)$

$$
\int f\left(\pi_{T_{2}}(\omega)\right) d P_{T}\left(d \omega / \pi_{T_{1}}(\omega)=\omega^{\prime}\right)
$$

depends continuously on $\omega^{\prime}$. By Theorem 2.2 , this equals

$$
\begin{aligned}
\left(\sum_{m=0}^{\infty} \int_{\mathcal{L}_{T \backslash T_{1}, m}^{0}} \frac{1}{m !} d^{m} \mu(\ell) \Sigma_{\omega^{\prime}} f\left(\pi_{T_{2}}(\omega)\right) e^{-F(\omega)}\right) \\
/\left(\sum_{m=0}^{\infty} \int_{\mathcal{L}_{T \backslash T_{1}, m}^{0}} \frac{1}{m !} d^{m} \mu(\ell) \Sigma_{\omega^{\prime}} e^{-F(\omega)}\right)
\end{aligned}
$$

when $\Sigma_{\omega^{\prime}}$ denotes the summation over $\omega$ in $\Omega_{T}\left((\ell)_{n} \cup \tilde{L}\left(\omega^{\prime}\right)\right)$ compatible with $\omega^{\prime}$. By the additivity of $F$, this is seen to equal

$$
\begin{array}{r}
\left(\sum_{m=0}^{\infty} \int_{\mathcal{L}_{T \backslash T_{1}, m}^{0}} \frac{1}{m !} d^{m} \mu(\ell) \Sigma^{\prime} f\left(\pi_{T_{2}}(\omega)\right) e^{-F\left(\pi_{T_{2}}(\omega)\right)}\right) \\
/\left(\sum_{m=0}^{\infty} \int_{\mathcal{L}_{T \backslash T_{1}, m}^{0}} \frac{1}{m !} d^{m} \mu(\ell) \Sigma^{\prime} e^{-F\left(\pi_{T_{2}}(\omega)\right)}\right)
\end{array}
$$

where $\Sigma^{\prime}$ denotes summation over $\pi_{T_{2}}(\omega)$ in $\Omega_{T_{2}}\left((\ell)_{n}\right)$ compatible with $\omega^{\prime}$. Then it suffices to prove that the last expression above depends continuously on $\omega^{\prime}$.

Proof: (Sketch) Let $\eta, \epsilon, \epsilon^{\prime}, \delta>0, N \geq 1, \tilde{\omega} \in \Omega_{T_{1}}$ and $D=\left\{\omega \in \Omega_{T_{3}} \mid \operatorname{sep}(\omega) \geq \delta, N(\omega) \leq N\right.$,

$\tilde{\omega}, \omega$ are compatible $\}.$

It is easy to see that $D$ is relatively sequentially compact in our topology on $\Omega_{T_{3}}$. Keep $\tilde{\omega}$ fixed henceforth and let $\bar{\omega} \in \Omega_{T_{1}}$ be such that $d(\operatorname{tr}(\tilde{\omega}), \operatorname{tr}(\bar{\omega}))<\epsilon$. Pick $\delta>0$ small enough and $N \geq 1$ large enough such that

$$
P\left(\pi_{T_{2}}(\omega) \in D / \operatorname{tr}\left(\pi_{T_{1}}(\omega)\right)=\operatorname{tr}(\tilde{\omega})\right)>1-\eta .
$$

Given $\omega^{\prime} \in D$, construct $\omega^{\prime \prime} \in \Omega_{T_{3}}$ compatible with $\bar{\omega}$ as follows: Let

$$
\begin{aligned}
& \operatorname{tr}(\tilde{\omega})=\left(i_{1}, x_{1}, r_{1}, \cdots, i_{n+1}\right) \\
& \operatorname{tr}(\bar{\omega})=\left(i_{1}, \bar{x}_{1}, \bar{v}_{1}, \cdots, i_{n+1}\right) .
\end{aligned}
$$




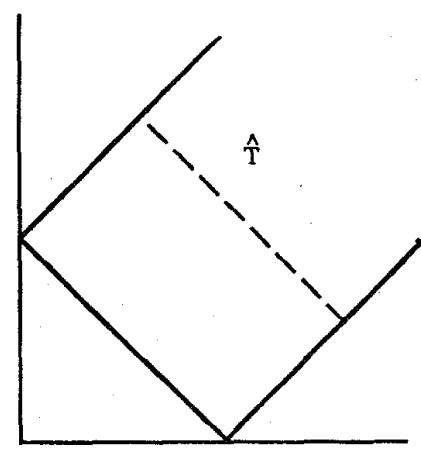

Fig. 11. Redrawing of domain $T$ in accordance with Fig. 2.

For each $k, 1 \leq k \leq n$, start a particle at $\bar{x}_{k}$ with velocity $\bar{v}_{k}$ and environment $\left(i_{k}, i_{k+1}\right)$.

Let births of the type depicted in Figs. 3-5 take place for $\omega^{\prime \prime}$ in exactly the same manner as for $\omega^{\prime}$. The particle in $\omega^{\prime \prime}$ that started at $\left(\bar{x}_{i}, \bar{v}_{i}\right)$ undergoes the same sequence of events of the type depicted in Figs. 6 and 7 as the particle in $\omega^{\prime}$ that started at $\left(x_{i}, v_{i}\right)$, with exactly the same times of occurence and same angles at the nodes generated thereby. The same also holds for the corresponding pairs of newly born particles ( $a$ la Figs. 3-5) in $\omega^{\prime}$ and $\omega^{\prime \prime}$. Furthermore, events of the type depicted in Figs. 8 and 9 are in one-to-one correspondence in $\omega^{\prime}, \omega^{\prime \prime}$ and occur in the same order. Finally, $\omega^{\prime \prime}$ satisfies: if $\bar{\omega}^{\prime}$ (respectively, $\bar{\omega}^{\prime \prime}$ ) denotes $\left.\tilde{\omega} \cup \omega^{\prime}\right|_{T_{2}}$ (respectively, $\left.\bar{\omega} \cup \omega^{\prime \prime}\right|_{T_{2}}$ ), then

$$
\left|\exp \left(-F\left(\omega^{\prime}\right)\right)-\exp \left(-F\left(\omega^{\prime \prime}\right)\right)\right|<\epsilon^{\prime},\left|f\left(\bar{\omega}^{\prime}\right)-f\left(\bar{\omega}^{\prime \prime}\right)\right|<\epsilon^{\prime} .
$$

Of course, all this may not be possible, but for prescribed $\epsilon^{\prime}, \delta$ and $N$, it is possible for $\bar{\omega}$ in a sufficiently small neighborhood of $\tilde{\omega}$. Let $h$ denote the map $\omega^{\prime} \rightarrow \omega^{\prime \prime}$ and let $D^{\prime}=h(D)$. Then $h: D \rightarrow D^{\prime}$ is seen to be a continuous bijection. Now $(*)$ and $(t)$ together lead to

$$
\begin{aligned}
\mid P_{T}\left(\pi_{T_{3}}(\omega) \in D^{\prime} / \pi_{T_{1}}(\omega)=\bar{\omega}\right)- & P_{T}\left(\pi_{T_{3}}(\omega)\right. \\
& \left.\in D / \pi_{T_{1}}(\omega)=\tilde{\omega}\right) \mid<\eta / 2
\end{aligned}
$$

for sufficiently small $\epsilon^{\prime}$ and (correspondingly small) $\epsilon$. Hence

$$
P_{T}\left(\pi_{T_{3}}(\omega) \in D^{\prime} / \pi_{T_{3}}(\bar{\omega})=\bar{\omega}\right) \geq 1-\eta / 2
$$

Using $(*),(\dagger)$ once more, we have, for $\bar{\omega}$ in a sufficiently small neighborhood of $\tilde{\omega}$

$$
\begin{aligned}
& \mid \int f\left(\pi_{T_{2}}(\omega)\right) P_{T}\left(d \omega / \pi_{T_{1}}(\omega)=\bar{\omega}\right) \\
& \quad-\int f\left(\pi_{T_{2}}(\omega)\right) P_{T}\left(d \omega / \pi_{T_{1}}(\omega)=\tilde{\omega}\right) \mid \\
& \leq 2 \eta K+\mid \int_{D} f\left(\pi_{T_{2}}(\omega)\right) P_{T}\left(d \omega / \pi_{T_{1}}(\omega)=\bar{\omega}\right) \\
& \quad-\int_{D^{\prime}} f\left(\pi_{T_{2}}(\omega)\right) P_{T}\left(d \omega / \pi_{T_{1}}(\omega)=\bar{\omega}\right) \mid<3 \eta K
\end{aligned}
$$

where $K>0$ is a bound on $|f(\cdot)|$. The claim follows.

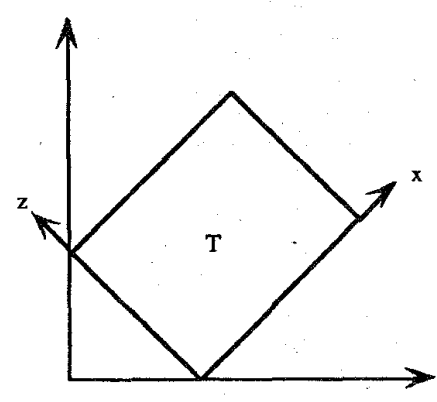

Fig. 12. Parameterization of $T$.

Pick $t>0$ large enough so that $\overline{T(0)} \cap \overline{T(t)}=\phi$. Let $\varphi_{t}: \Omega_{T(0)} \rightarrow \Omega_{T(t)}$ denote the map

$$
(s, y) \rightarrow(s+t \cos \theta, y+t \sin \theta)
$$

as before.

Lemma 4.3: For any open set $O \subset \Omega_{T}$ and $\omega \in \Omega_{T}$

$$
P\left(\xi_{t} \in O / \xi_{0}=\omega\right)>0 .
$$

Proof: It suffices to consider $O=$ an open neighborhood of $\bar{\omega} \in \Omega_{T}$. Let $C=$ the convex hull of $T(0)$ and $T(t)$. By introducing an appropriate number of births, deaths, branching, etc., in $C \backslash \overline{(T(0) \cup T(t))}$, we can always construct a valid trajectory $\eta$ of $\{x(\cdot)\}$ that restricts to $\omega$ on $T(0)$ and $\bar{\omega} \circ \varphi_{t}^{-1}$ on $T(t)$. Then from the particle dynamics described in the preceding section, it is clear that for any open set $A \subset \Omega_{C}$ containing $\eta$

$$
P_{C}\left(\pi_{T(t)}(\tilde{\omega}) \in A / \pi_{T(0)}(\tilde{\omega})=\omega\right)>0 .
$$

Pick $A$ such that $\pi_{T(t)}(A) \subset$ the image of $O$ under $\varphi_{t}$, to conclude.

Thus we have an $\Omega_{T}$-valued $R$-reversible ergodic process $\left\{\xi_{t}\right\}$ with invariant distribution $P_{T}$. One may also consider its discrete time version, i.e., a process $\xi_{n}^{\Delta}=\xi_{\Delta n}, n=$ $0, \pm 1, \cdots$, for some $\Delta>0$. This will be a discrete-time $R$-reversible ergodic process with invariant measure $P_{T}$.

We shall now use $\left\{\xi_{t}\right\}$ as a basis for constructing a discretetime reversible ergodic process $\left\{Z_{n}\right\}$ such that for fixed $n$, 

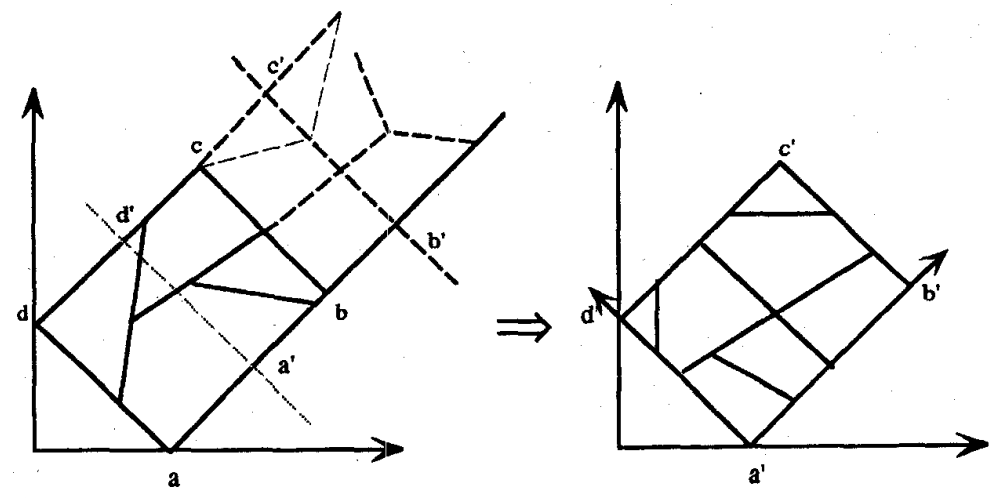

Fig. 13. Step in describing state transition for process $\xi_{t}$.

$Z_{n}$ is a PRF with potential $H$. For this purpose, introduce the following convention: Parametrize $T$ as

$$
T=\left\{(x, z) \mid 0<x<b_{1}, 0<z<b_{2}\right\}
$$

where $b_{1}, b_{2}$ are the lengths of the sides of $T$ (see Fig. 12). Let

$$
T_{p}=\left\{(x, z) \mid 0<x<b_{1} / 2,0<z<b_{2}\right\}
$$

and

$$
T_{s}=\left\{(x, z) \mid b_{1} / 2<x<b_{1}, 0<z<b_{2}\right\} .
$$

Given $\omega \in \Omega_{T}$ define $\omega_{p} \in \Omega_{T_{p}}$ and $\omega_{s} \in \Omega_{T_{s}}$ as the restrictions of $\omega$ to $T_{p}, T_{s}$, respectively, which we refer to as the prefix and the suffix of $\omega$. Given $\omega, \omega^{\prime} \in \Omega_{T}$, we say that $\omega, \omega^{\prime}$ are neighbors if and only if either $\omega_{p}^{\prime}=\omega_{s}$ or $\omega_{s}^{\prime}=\omega_{p}$. This is clearly a symmetric relation. Let

$$
N(\omega) \triangleq\{\text { neighbors of } \omega\} \subset \Omega_{T} .
$$

Suppose $Z_{n}=\omega$ for some $n$. The state transition at time $n$ is effected as follows: First pick one element from the set $\{p, s\}$ with equal probability. Suppose you get $s$. Let an independent copy of the process $x(\cdot)$ evolve conditioned on $\left.x(\cdot)\right|_{T}=\omega$. Let $\tilde{\omega}=x(\cdot)$ restricted to $T\left(b_{1} t / 2\right)$. Thus $\tilde{\omega} \in \Omega_{T\left(b_{1} t / 2\right)}$. Let $\omega^{\prime}=\varphi_{s}^{-1}(\tilde{\omega}) \in \Omega_{T}$, where $s=b_{1} t / 2$. Set $Z_{n+1}=\omega^{\prime}$ with probability $\exp \left[-\left(G\left(\omega^{\prime}\right)-G(\omega)\right)^{+}\right)$and $=\omega$ with probability $1-\exp \left(-\left(G\left(\omega^{\prime}\right)-G(\omega)\right)^{+}\right)$where $G=H-F$. Note that $\omega_{p}^{\prime}=\omega_{s}$ and thus $\omega^{\prime} \in N(\omega)$ (Fig. 13). If one picks $p$ instead of $s$ in the first step, the procedure is similar except that one evolves $x(\cdot)$ in reversed time, leading to $\omega_{s}^{\prime}=\omega_{p}$. Then $\left\{Z_{n}\right\}$ is an $\Omega_{T}$-valued Markov process whose transition probability is given by

$$
\begin{aligned}
P & \left(Z_{n+1} \in[\tilde{\omega}, \tilde{\omega}+d \tilde{\omega}] / Z_{n}=\bar{\omega}\right) \\
& =\frac{1}{2}\left(P _ { T } \left(\pi_{T_{s}}(\omega) \in[\tilde{\omega}, \tilde{\omega}+d \tilde{\omega}] / \pi_{T_{p}}(\omega)\right.\right. \\
& \left.=\pi_{T_{p}}(\bar{\omega})\right)+P_{T}\left(\pi_{T_{p}}(\omega) \in[\tilde{\omega}, \tilde{\omega}+d \tilde{\omega}] / \pi_{T_{s}}(\omega)\right. \\
& \left.\left.=\pi_{T_{s}}(\bar{\omega})\right)\right) \exp \left(-(G(\tilde{\omega})-G(\bar{\omega}))^{+}\right) d \tilde{\omega}
\end{aligned}
$$

for $\tilde{\omega} \neq \bar{\omega}$ and

$$
P\left(Z_{n+1}=\bar{\omega} / Z_{n}=\bar{\omega}\right)=1-P\left(Z_{n+1} \neq \bar{\omega} / z_{n}=\bar{\omega}\right)
$$

where the rightmost quantity is obtained by integrating the right-hand side of the preceding equation over $\{\tilde{\omega} \mid \tilde{\omega} \neq \bar{\omega}\}$.
Theorem 4.2: $\left\{Z_{n}\right\}$ is a reversible ergodic process with invariant measure $P_{T, H, \mu}$.

Proof: Let $\xi_{n}=\xi_{n}^{\Delta}, n=0, \pm 1, \pm 2, \cdots$, for $\Delta=$ $\left(b_{1} \cos \theta\right) / 2$. Let $\nu^{+}\left(\omega, d \omega^{\prime}\right), \nu^{-}\left(\omega, d \omega^{\prime}\right), \tilde{\nu}\left(\omega, d \omega^{\prime}\right)$ denote the transition probability measures for $\left\{\xi_{n}\right\},\left\{\xi_{-n}\right\},\left\{Z_{n}\right\}$, respectively. Then

$$
\begin{aligned}
\tilde{\nu}\left(\omega, d \omega_{1}\right)= & \frac{1}{2} e^{-\left(G\left(\omega_{1}\right)-G(\omega)\right)^{+}}\left(\nu^{+}\left(\omega, d \omega_{1}\right)+\nu^{-}\left(\omega, d \omega_{1}\right)\right) \\
& +g(\omega) \delta_{\omega}\left(d \omega_{1}\right)
\end{aligned}
$$

where $\delta_{\omega}(\cdot)$ is the Dirac measure at $\omega$ and

$$
\begin{aligned}
1-g(\omega)=\frac{1}{2} & \int_{\Omega_{T}} e^{-\left(G\left(\omega_{1}\right)-G(\omega)\right)^{+}} \\
& \cdot\left(\nu^{+}\left(\omega, d \omega_{1}\right)+\nu^{-}\left(\omega, d \omega_{1}\right)\right) .
\end{aligned}
$$

Let $\dot{\eta}=P_{T, F, \mu}\left(=P_{T}\right)$ and $\tilde{\eta}=P_{T, H, \mu}$. Then

$$
\tilde{\eta}(d \omega)=e^{-G(\omega)} \eta(d \omega)
$$

We need to show that

$$
\tilde{\eta}(d \omega) \tilde{\nu}\left(\omega, d \omega^{\prime}\right)=\tilde{\eta}\left(d \omega^{\prime}\right) \tilde{\nu}\left(\omega^{\prime}, d \omega\right) .
$$

The left-hand and the right-hand sides, respectively, equal

$$
\begin{array}{r}
\frac{1}{2} \eta(d \omega) e^{-G(\omega)} e^{-\left(G\left(\omega^{\prime}\right)-G(\omega)\right)^{+}}\left(\nu^{+}\left(\omega, d \omega^{\prime}\right)+\nu^{-}\left(\omega, d \omega^{\prime}\right)\right) \\
+\eta(d \omega) e^{-G(\omega)} g(\omega) \delta_{\omega}\left(d \omega^{\prime}\right)
\end{array}
$$

and

$\frac{1}{2} \eta\left(d \omega^{\prime}\right) e^{-G\left(\omega^{\prime}\right)} e^{-\left(G(\omega)-G\left(\omega^{\prime}\right)\right)^{+}}\left(\nu^{+}\left(\omega^{\prime}, d \omega\right)+\nu^{-}\left(\omega^{\prime}, d \omega\right)\right)$

$$
+\eta\left(d \omega^{\prime}\right) e^{-G\left(\omega^{\prime}\right)} g\left(\omega^{\prime}\right) \delta_{\omega^{\prime}}(d \omega) .
$$

It is easily checked that $\eta(d \omega) \exp (-G(\omega)) g(\omega) \delta_{\omega}\left(d \omega^{\prime}\right)$ and $\eta\left(d \omega^{\prime}\right) \exp \left(-G\left(\omega^{\prime}\right)\right) g\left(\omega^{\prime}\right) \delta_{\omega^{\prime}}(d \omega)$ represent the same measure concentrated on the diagonal $\left\{\omega=\omega^{\prime}\right\}$. Thus we only need to verify that the first terms of the above expressions match. Consider the case $G\left(\omega^{\prime}\right) \geq G(\omega)$. (The reverse case follows by a symmetric argument.) Then we are reduced to verifying

$$
\begin{aligned}
\eta(d \omega)\left(\nu^{+}\left(\omega, d \omega^{\prime}\right)+\nu^{-}\right. & \left.\left(\omega, d \omega^{\prime}\right)\right) \\
& =\eta\left(d \omega^{\prime}\right)\left(\nu^{+}\left(\omega^{\prime}, d \omega\right)+\nu^{-}\left(\omega^{\prime}, d \omega\right)\right)
\end{aligned}
$$


Since $\eta$ is the invariant measure for $\left\{\xi_{n}\right\}$, we have

$$
\eta(d \omega) \nu^{+}\left(\omega, d \omega^{\prime}\right)=\eta\left(d \omega^{\prime}\right) \nu^{-}\left(\omega^{\prime}, d \omega\right) .
$$

This completes the proof of the fact that $\left\{Z_{n}\right\}$ is stationaryreversible when the law of $Z_{0}$ is $\tilde{\eta}$. Ergodicity follows by arguments analogous to those used for proving Theorem 3.1.

\section{Examples of $H$ :}

1) Consider the PRF given by $P_{T}$ observed at points $\left\{t_{1}, \cdots, t_{n}\right\} \subset T$ through a channel with distortion and noise, modeled as follows: We have observations $y_{i}=f\left(\omega\left(t_{i}\right)\right)+\beta_{i}, 1 \leq i \leq n$, for some function $f: \Omega_{T} \rightarrow R$ and i.i.d. $N\left(\overline{0}, \sigma^{2}\right)$ random viarables $\beta_{1}, \cdots, \beta_{n}$. The posterior distribution of the PRF given these observations then corresponds to a PRF with distribution $P_{T, H, \mu}$ where [5], [6]

$$
H(\omega)=F(\omega)+\sum_{i=1}^{n} f^{2}\left(\omega\left(t_{i}\right)\right) / 2 \sigma^{2}-\sum_{i=1}^{n} y_{i} f\left(\omega\left(t_{i}\right)\right) / \sigma^{2}
$$

2) An alternative model of observations is [5]: We observe an inhomogeneous Poisson point process on $T$ generated by $\omega$ with spatial intensity $f(\omega(t))$ at point $t$. The posterior distribution now corresponds to

$H(\omega)=F(\omega)+\int_{T} f(\omega(t)) d t-\int_{T} \log f(\omega(t)) \Lambda(d t)$

where $\Lambda$ is the counting measure for the observed point process [5].

3) We may take $H=F+G$ where $G(\omega)=$ the sum of angles (in absolute value) between any two straight-line segments in $\partial \omega$ that meet each other. This is in the spirit of the "total turn" considered in [7].

Note that each $H$ above is additive and thus $P_{T, H, \mu}$ is a Markov random field by the arguments of [2, sec. 8].

The process $\left\{Z_{n}\right\}$ proposed above has much simpler dynamics compared to the processes proposed in [4]-[6]. In the next section, we consider a variant that permits segmentations with curved boundaries.

\section{EXTENSIONS TO GPRF}

This section extends some of the foregoing to "Generalized Polygonal Random Fields" (GPRF) which have polygonal-like realizations, but with curved boundaries. We begin with some preliminaries.

To each $x \in \mathcal{R}^{2}$, attach a set $C_{x}$ of non-self-intersecting $C^{1}$ curves through $x$ satisfying

1) Each $c \in C_{x}$ admits a parametrization $t \in \mathcal{R} \rightarrow z_{c}(t)=$ $\left[x_{c}(t), y_{c}(t)\right]$ such that $x_{c}(\cdot), y_{c}(\cdot) \in C^{1}, z_{c}(0)=x$. We write $c \sim z_{c}(\cdot)$. Without loss of generality, we may and do assume that $\dot{x}_{c}(t)^{2}+\dot{y}_{c}(t)^{2}=1 \forall t$. Also, $\left\{z_{c}(\cdot) \mid c \in C_{x}\right\}$ is assumed to be compact under the topology of uniform convergence on compacts.

2) For any bounded open $A \subset \mathcal{R}^{2}$ with $x \in A$

$$
\sup \left\{|t| \mid z_{c}(t) \in A\right\}<\infty \text {. }
$$

3) If $c \in C_{x}$ and $c^{\prime}$ is obtained by rotating $c$ around $x$, then $c^{\prime} \in C_{x}$. (This operation will be called rotation.)

4) If $c \in C_{x}$, then $c^{\prime} \in C_{x}$ for $c^{\prime} \sim z_{c}(\tau+\cdot)-z_{c}(\tau)+x, \tau \in$ $\mathcal{R}$. (This operation will be called time shift.)

5) If $c \in C_{x}$, then $c^{\prime} \in C_{x}$, when $z_{c^{\prime}}(t)=z_{c}(-t)$. (This operation will be called time reversal.)

6) If $\theta \in \mathcal{R}^{2}$ denotes the origin

$$
C_{x}=\left\{c \mid z_{c}(\cdot)=x+z_{c^{\prime}}(\cdot) \text { for some } c^{\prime} \in C_{\theta}\right\} .
$$

7) If $c \in C_{x}, c^{\prime} \in C_{y}$ satisfy $z_{c}(t)=z_{c^{\prime}}(\tau+t)$ for $t \in$ $(a, b)$, for some $a<b$ and $\tau \in \mathcal{R}$, then $z_{c}(\cdot)=z_{c^{\prime}}(\tau+\cdot)$.

For $A \subset \mathcal{R}^{2}$, set $C_{A}=\bigcup_{x \in A} C_{x}$.

Remark 5.1: If for $c \in C_{x}, z_{c}(\cdot)$ is viewed as the trajectory of a particle starting at $x, 2$ ) implies that the particle exits from any finite domain in finite time. 6) says the $C_{x}$ is obtained from $C_{\theta}$ by translation, so it suffices to prescribe $C_{\theta}$.7) says that if two trajectories agree on a nonempty open interval, one must be a time shift of the other. $\overline{\mathrm{By}} 3$ ) -5$), C_{x}$ is closed under rotation, time shift, and time reversal.

Example 5.1: Let $\hat{C}_{\theta}$ be a finite collection of curves $c_{1}, \cdots, c_{n}$ passing through $\theta$ such that $z_{c_{i}}(t)=\left[t, f_{i}(t)\right]$ where $t \rightarrow f_{i}(t)$ are periodic with a common period $\tau$ and no piece of any one of the curves or any of its rotations or translations coincides with any other of these curves on some interval. Let $C_{\theta}=$ all curves obtained from $\hat{C}_{\theta}$ by rotation, time shift, or time reversal $\} . C_{x}, x \in \mathcal{R}^{2}$ are now automatically specified through 6).

Typically one expects to obtain $C_{\theta}$ from a "core" $\hat{C}_{\theta}$ by the above procedure. As we shall be interested in $C_{T}$ for a rectangle $T$, the above example may often provide a sufficiently rich class in applications for suitable choices of $n,\left\{c_{1}, \cdots, c_{n}\right\}$ and with $\tau \gg$ diameter $(T)$. It has the advantage of easy parametrization.

Let $\zeta_{\theta}$ denote a probability measure on $C_{\theta}$ which is invariant under rotation, time shift, and time reversal. The existence of a probability measure that is invariant under rotation and time shift is guaranteed by elementary ergodic theory. It may be rendered invariant under time reversal by taking its image under time reversal and then taking the average of the two. We assume that support $\left(\zeta_{\theta}\right)=C_{\theta}$. (If not, it is equivalent to considering a smaller $C_{\theta}$, viz., support $\left(\zeta_{\theta}\right)$.) Let $\zeta_{x}$ denote the probability measure on $C_{x}$ obtained as the image of $\zeta_{\theta}$ under the map $c \in C_{\theta} \rightarrow x+c \in C_{x}$.

Let $T \subset \mathcal{R}^{2}$ be a prescribed rectangle as before. By a "raw image" on $T$, we mean $T$ endowed with a finite collection of finite curves, each of them a segment of some element of $C_{T}$. We shall now construct a probability measure on $I_{R}=$ the set of all raw images on $T$. This is done in the following steps:

\section{Procedure 1:}

i) Generate random points in $T$ according to a Poisson point process with intensity $\bar{\lambda}$

ii) From each point $x$ obtained above, pick a random curve

$$
c \sim z_{c}(\cdot)=\left[x_{c}(\cdot), y_{c}(\cdot)\right] \in C_{x}
$$

according to $\zeta_{x}$. 
iii) Initiate a particle at each $x$ with trajectory $t \rightarrow z_{c}(t)$, $t \geq 0$, and with extinction time exponentially distributed with mean 1 . Extinction times of distinct particles are independent.

iv) Draw the traces of their trajectories till the extinction time or the first time they hit $\partial T$, whichever occurs first, thus obtaining a finite segment of the corresponding curve.

This clearly gives an isotropic probability measure on $I_{R}$, viz., the law of the raw image generated by the above procedure.

Given a raw image $\gamma \in I_{R}$, let $D(\gamma)$ denote the set of curve segments that constitutes $\gamma$ and $G(\gamma)$ their union. Let $A \subset T$ be a connected component of $T \backslash G(\gamma)$. Then $\partial A \subset G(\gamma) \cup \partial T$. We can write $\partial A=\partial_{1} A+\partial_{2} A$ where $\partial_{1} A$ is that part of $\partial A$ which is also a part of the boundary of some other connected component of $T \backslash G(\gamma)$ or of $\partial T$, and $\partial_{2} A=$ $\partial A \backslash \partial_{1} A$. Let $A^{\prime}=$ interior of $A \cup \partial_{2} A$. Then $\partial A^{\prime}=\partial_{1} A$. A set $A^{\prime}$ thus obtained will be called a piece of $\gamma$. In Fig. 14, for example, if $A$ is the interior of the region bounded by the contour $a b c d$ with the curve ef removed, then $A^{\prime}$ is the entire interior of the same region. Let $G_{b}(\gamma) \subset G(\gamma)$ denote the union of all $\partial A^{\prime}$ such that $A^{\prime}$ is a piece of $\gamma$. For each $c \in D(\gamma)$ parametrized as, say, $c=\{z(t) \mid a \leq t \leq b\}$, define $b(c) \subset c$ as follows. If $c \cap G_{b}(\gamma) \neq \phi, b(c)=\left\{z(t) \mid a^{\prime} \leq t \leq b^{\prime}\right\}$ with $a \leq a^{\prime}, b \geq b^{\prime}$, such that $b(c)$ is the minimal such set containing $c \cap G_{b}(\gamma)$. If $c \cap G_{b}(\gamma)=\phi, b(c)=\phi$. Define the "trimming operator" $\operatorname{Tr}: I_{R} \rightarrow I_{R}$ to be the map that maps $\gamma \in I_{R}$ to its "trimmed version" $\gamma^{\prime} \in I_{R}$ obtained by replacing each $c \in D(\gamma)$ by $b(c)$. Fig. 15 shows a raw image and its trimmed version. We shall denote by $I_{T}$ the set of trimmed images, i.e., the range of Tr. By a proper image (or simply an image) we mean a map $\omega: T \rightarrow J \cup\left\{j^{*}\right\}, J$ being a finite set of colors as before and $j^{*} \notin J$ another distinguished color, such that the following hold: These exist $\gamma(\omega) \in I_{T}$ such that $\omega$ is constant and equal to an element of $J$ on each piece of $\gamma(\omega), \omega=j^{*}$ on $\cup_{c \in D\left(\gamma_{(\omega)}\right)} b(c)$. Thus $\partial \omega=G_{b}(\gamma(\omega))$, where $\partial \omega=$ the set of points of discontinuities of $\omega$. Let $I$ denote the set of images. Note that unlike in the case of PRF's, we are allowing "internal" discontinuities that lie in the interior of a piece and not on its boundary. (For example, if $\gamma(\omega)$ is as in Fig. 15(b), then $\omega$ will have the same color on either side of the segment $a b$, but a different color on it.) Conversely, given $\gamma \in I_{T}$, define

$$
\Omega(\gamma)=\{\omega \in I \mid \partial \omega=G(\gamma)\}
$$

and $\lambda(\gamma)=|\Omega(\gamma)|$. In the foregoing, we have a procedure for generating a random $\gamma \in I_{T}$ (viz., generate a random element of $I_{R}$ by Procedure 1 and trim it). Given this $\gamma$, we may generate a random $\omega \in I$ by picking any element of $\Omega(\gamma)$ with equal probability $(=1 / \lambda(\gamma))$. Let $P_{T}=$ the probability measure on $I$ induced by the random sample thereof generated as above, where we endow $I$ with the Borel $\sigma$-field corresponding to its topology defined analogously as for $\Omega_{T}$. We call $P_{T}$ a Generalized Polygonal Random. Field (GPRF) on $T$.

Define on $C_{T}$ an equivalence relation " $\approx$ " by: $c \approx c^{\prime}$ if $c^{\prime}$ is a time shift of $c$. Let $\hat{C}_{T}$ denote the set of equivalence

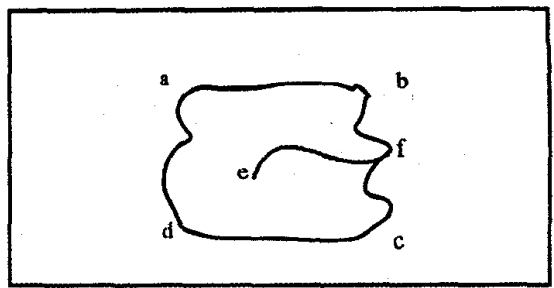

Fig. 14. The set $A^{\prime}=$ the region bounded by the contour $a b c d$ is a piece of $\gamma$.

classes thus obtained and

$$
\mathcal{C}_{n}=\left\{\mathcal{C} \subset \hat{C}_{T}|| \mathcal{C} \mid=n\right\}, \quad n=0,1,2, \cdots
$$

Let $\mu_{n}$ denote the probability measure on $\mathcal{C}_{n}$ induced by steps i) and iii) of Procedure 1, conditioned on $n$ curves being picked by the procedure. Probability of the latter event is

$$
(\bar{\lambda}|T|)^{n} \exp (-\bar{\lambda}|T|) / n !
$$

Clearly, $\mu_{n}$ is isotropic for each $n$. For $\eta \in \mathcal{C}_{n}, n \geq 0$, let

$I_{T}(\eta)=\left\{\omega \in I \mid G_{b}(\gamma(\omega)) \subset \eta, G_{b}(\gamma(\omega)) \not \subset \eta^{\prime}\right.$ for any proper subset $\eta^{\prime}$ of $\eta$.

Note that this is a finite set. As before, $L(\omega)=$ the total length of $\partial \omega$ for $\omega \in I$.

Theorem 5.I: The GPRF $P_{T}$ obtained above is an isotropic Markov random field given by

$$
\begin{aligned}
P_{T}(A)= & \left(\sum_{n=0}^{\infty} \frac{(\bar{\lambda}|T|)^{n}}{n !} e^{-\bar{\lambda}|T|} \int_{\mathcal{C}_{n}} \mu_{n}(d \eta)\right. \\
& \left.\times \sum_{I_{T}(\eta) \cap A} e^{-(L(\omega)+\log [\lambda(\gamma(\omega))])}\right) / Z_{T}
\end{aligned}
$$

where $Z_{T}$ is the normalizing constant:

Proof: Isotropy of $P_{T}$ follows from its construction. Now the probability that Procedure 1 picks $n$ curves $c_{1}, \cdots, c_{n}$ in $[\eta, \eta+d \eta] \subset \mathcal{C}_{n}$ and the independent system of particles planted one each on these survives for larger than $\ell_{1}, \cdots, \ell_{n}$ (respectively) time units (call this entire event $Q$ ) is

$$
\frac{(\bar{\lambda}|T|)^{n}}{n !} e^{-\bar{\lambda}|T|} \mu_{n}(d \eta) e^{-\left(\ell_{1}+\cdots+\ell_{n}\right)}
$$

The traces left by these particles need not, however, lead to a legal element of $I_{T}$. Hence the probability of obtaining an element $\gamma(\omega) \in I_{T}(\eta)$ thus is the probability of $Q$ conditioned on the particle traces constituting an element of $I_{T}$. This is

$$
\sum_{n=0}^{\infty} \frac{(\bar{\lambda}|T|)^{n}}{n !} e^{-\bar{\lambda}(T)} \mu_{n}(d \eta) I\left\{\gamma(\omega) \in I_{T}(\eta)\right\} e^{-L(\omega)} / Z_{T}
$$

with

$Z_{T}=\sum_{n=0}^{\infty} \frac{(\bar{\lambda}|T|)^{n}}{n !} e^{-\bar{\lambda}|T|} \int_{\mathcal{C}_{n}} \mu_{n}(d \eta) I\left\{\gamma(\omega) \in I_{T}(\eta)\right\} e^{-L(\omega)}$.

Given $\gamma(\omega)$, a candidate $\omega$ is picked by choosing a coloring with probability

$$
1 / \lambda(\gamma(\omega))=\exp (-\log \lambda(\gamma(\omega)))
$$




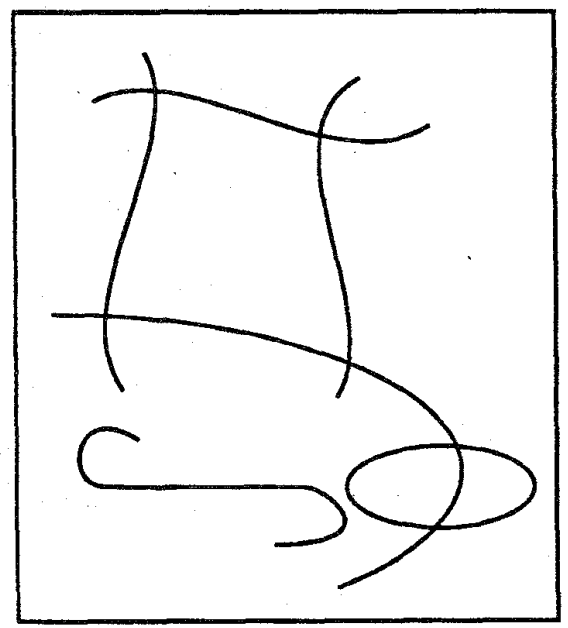

(a)

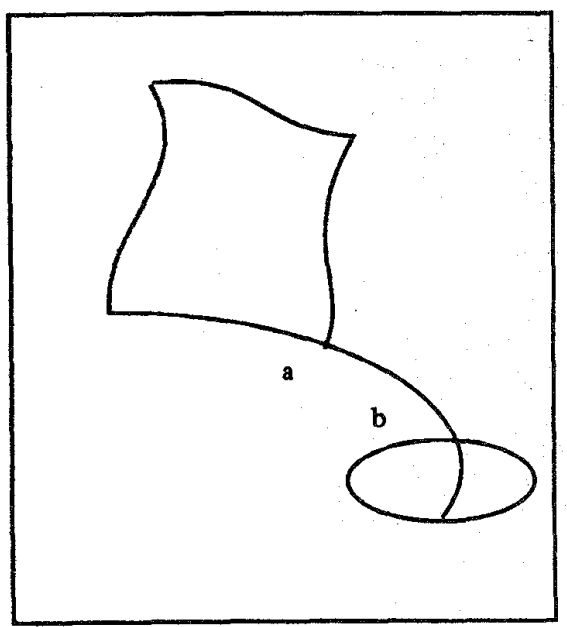

(b)

Fig. 15. Raw image and its trimmed version. (a) Raw. (b) Trimmed.

This completes the derivation of (5.1). The Markov property can be proved as follows: Let $T=S \cup U, S, U$ open. Consider $\gamma \in I_{\dot{T}}$ generated by Procedure 1. Then this procedure implies that the conditional statistics of $\pi_{U}(\gamma)$ given $\pi_{S}(\gamma)=\xi$ (say) may be simulated as follows: Generate random points in $U \backslash S$ according to a Poisson point process with intensity $\bar{\lambda}$ and follow ii)-iv) of Procedure 1. Also, extend those curves in $\xi$ that hit $\partial S \cap U$ and can be extended into $U \backslash S$, as in iii) of Procedure 1. Trim the resulting image. Accept it if it restricts to $\xi$ on $S$, otherwise reject and repeat the procedure. Now it is clear that in the above, one could replace $T$ by $U, S$ by $U \cap S$, and $\xi$ by its restriction to $U \cap S$ to obtain the same statistics for $\pi_{U}(\gamma)$. This is because $T=U \cup S$ and thus any curve in $\gamma$ that straddles both $S$ and $U$ must pass through $S \cap U$. Markov property follows. (A more formal proof could be given along the lines of $[2, \mathrm{sec} .8]$.) Now to prove that it is preserved in our passage from $I_{T}$ to $I$, we only need verify that the "potential" $\log \lambda(\gamma(\omega))$ is additive. The event of picking a random coloring of $\gamma(\omega) \in I_{T}$ can be viewed as taking place in two steps: First one picks a coloring for the restriction of $\gamma(\omega)$ to $S$ (denoted $\gamma_{S}(\omega) \in I_{S}$ ) according to uniform probability $1 / \lambda\left(\gamma_{S}(\omega)\right)$. Let $\gamma_{U}(\omega) \in I_{U}, \gamma_{S \cap U}(\omega) \in I_{S \cap U}$ denote the restrictions of $\gamma(\omega)$ to $U$ and $S \cap U$, resectively, and $\lambda\left(\gamma_{U}(\omega) / \beta\right)$ the number of possible colorings of $\gamma_{U}(\omega)$ compatible with the coloring of $\gamma_{S \cap U}(\omega)$ given by $\beta=$ the coloring it inherited from $\gamma_{S}(\omega)$. The second step is to color $\gamma_{U}(\omega)$ by picking a random coloring from those compatible with $\gamma_{S \cap U}(\omega)=\beta$ with equal probability, i.e., with probability $\lambda\left(\gamma_{U}(\omega) / \beta\right)^{-1}$. Then

$$
1 / \lambda(\gamma(\omega))=\left(1 / \lambda\left(\gamma_{S}(\omega)\right)\right)\left(1 / \lambda\left(\gamma_{U}(\omega) / \beta\right)\right)
$$

and

$$
\log \lambda(\gamma(\omega))=\log \lambda\left(\gamma_{S}(\omega)\right)+\log \lambda\left(\gamma_{U}(\omega) / \beta\right) .
$$

Thus $\log \lambda(\gamma(\omega))$ is additive.

Remark 5.2: An important limitation of the above theorem, in contrast to the corresponding result for PRF's, is that we do not claim the family $P_{T}, T \in \mathcal{G}_{0}$, to be consistent. In order to achieve consistency, it is clear that one will have to allow the particle trajectories that hit the boundary re-enter if they do so before the extinction time. But then a curve may contribute to the image more than one segment separated in space (i.e., with strictly positive distance from each other). The Markov property cannot hold in such a situation.

The next task is to generate an $I$-valued reversible ergodic Markov process $\left\{Z_{n}\right\}$ whose law at any time instant is

$$
\tilde{P}_{T}(d \omega)=\alpha P_{T}(d \omega) \exp (-G(\omega))
$$

for an additve $G: I \rightarrow[0, \infty], \alpha$ being the normalization constant. We mimick closely the earlier procedure for the PRF's, as described below: Define $T_{p}, T_{s}$ and the prefix $\omega_{p}$ and suffix $\omega_{s}$ of an image $\omega \in I$ the same way as we did for the PRF's.

\section{Procedure 2:}

Let $Z_{n}=\omega$.

i) Pick one element of $\{p, s\}$ with equal probability (say, s).

ii) Construct $\omega^{\prime} \in I$ as follows:

a) Set $\omega_{p}^{\prime}=\omega_{s}$ (see Fig. 16).

b) In $T_{s}$, pick $m$ (say) points according to a Poisson point process with rate $\bar{\lambda}$. At each point, pick a random curve as in Procedure 1, ii).

c) From each point picked in b) and each point on ef where a trajectory from $T_{p}$ hits ef, start a particle with exponential lifetime and unit speed along the corresponding curve. (In the latter case, the motion should be toward the interior of $T_{s}$ ). Trace its trajectory till extinction or till it hits $\partial T$, whichever comes first.

d) Trim the resultant raw image.

e) If the trimmed image does not restrict to $\gamma\left(\omega_{s}\right)$ on $T_{p}$, then reject those trajectories that led to the alterations of the trimmed image on $T_{p}$ and replace 


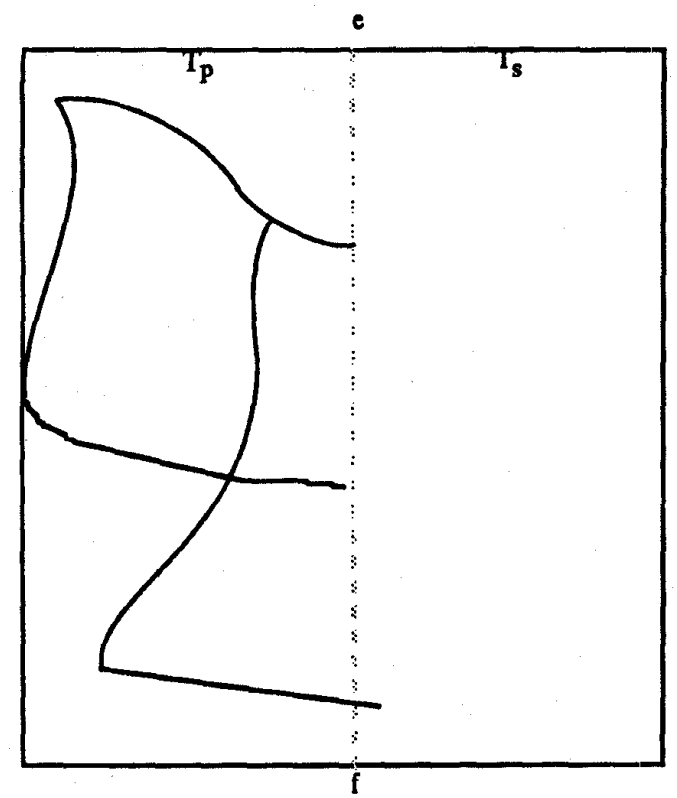

Fig. 16. Step in the construction of reversible process for polygon with curved boundaries.

them by new independently generated trajectories from the same initial points. Trim again.

f) Repeat till the trimmed image is consistant with $\gamma\left(\omega_{s}\right)$ on $T_{p}$

g) Color the trimmed image on $T_{s}$ by sampling uniformly from all colorings thereof that are compatible with the coloring on $T_{p}$. The resultant image on $T$ is the desired $\omega^{\prime}$. (In practice, this step calls for a good graph coloring heuristic.)

iii) Set $Z_{n+1}=\omega^{\prime}$ with probability $\exp \left(-\left(G\left(\omega^{\prime}\right)-\right.\right.$ $G(\omega))^{+}$) and $=\omega$ with the remaining probability.

Theorem 5.2: $\left\{Z_{n}\right\}$ is a reversible ergodic Markov process with stationary distribution $\tilde{P}_{T}$.

This can be proved by adapting the proofs of the corresponding results for PRF's. We omit the lengthy details. As for PRF, we may choose $G$ so as to incorporate an observationdependent term for Bayesian analysis or to incorporate extra "costs" such as the "total turn" discussed in [7].

A "greedy" heuristic for step (Procedure iig) above is as follows: identify the "uncolored" image with a planar graph by identifying each piece of it with a node, with two nodes connected by an edge if and only if the corresponding pieces are adjacent (i.e., their boundaries intersect). Rank the nodes in the decreasing order of their degrees. Color the top node (i.e., the corresponding piece) by an element of $J$ picked with uniform probability. Color the nodes in decreasing order, picking a color at each step uniformly from the admissible colors at that node. If a node is encountered for which there is no color left admissible, restart the whole procedure. Repeat till a complete coloring is found. (A color is "admissible" if it has not been already used to color a neighboring node.)

\section{APPENDIX}

We summarize here from [2] the conditions on coefficients featuring in the definition of $F$. Here $\left[\left[p_{i j}\right]\right], i, j \in J$, is a stochastic matrix with $p_{i j}=p_{j i}, p_{i i}=0, i, j \in J$.

$$
\begin{aligned}
& p_{i j}=a(i, j)=a(j, i), b(i, j)=b(j, i) . \\
& c(i ; j, k)=c(i ; k, j) . \\
& d(i, j, k, m)=d(j, k, m, i)=d(k, j, i, m) . \\
& b(i, j)+\sum_{k \neq j} d(i, j, k, j) p_{j k}^{2}=1 \\
& (c(i ; j, k)+c(j ; i, k)) p_{i j}+\sum_{m \neq i, j} d(i, k, j, m) \\
& \sum_{k \neq i, j}(c(i ; j, k)-c(j ; i, k)) p_{i k} p_{j k}=0 . \\
& e(i, j)=b(i, j) p_{i j}+\sum_{k \neq i, j} c(i ; j, k) p_{i j} p_{j k} . \\
& f(i)=c_{1} \sum_{j \neq i} p_{i j}^{2} b(i, j),
\end{aligned}
$$$$
(c(i ; j, k)+c(j ; i, k)) p_{i j}+\sum_{m \neq i, j} d(i, k, j, m) p_{j m} p_{i m}=1
$$

with

$$
c_{i}=\iint_{\mathcal{R}^{2}}|u-v| V(d u) V(d v)
$$

\section{ACKNOWLEDGMENT}

The authors wish to thank the referee for a very careful reading of the paper.

\section{REFERENCES}

[1] T. Arak, "On Markovian random fields with finite number of values," in 4th USSR-Japan Symp. on Probability Theory and Mathematical Statistics (Abstracts of Communications), Tbilisi, Georgia, 1982.

[2] T. Arak and D. Surgailis, "Markov fields with polygonal realizations," Prob. Theory Related Fields, vol. 80, pp. 543-579, 1989.

[3] ___ "Consistent polygonal fields," Prob. Theory Related Fields, vol. 89 , pp. $319-346,1991$.

[4] P. Clifford, "Markov random fields in statistics," in Disorder in Physical Systems, G. R. Grimmett and D. J. A. Welsh, Eds. Oxford, UK: Oxford Univ. Press, 1990, pp. 19-32.

[5] P. Clifford and R. D. Middleton, "Reconstruction of polygonal images," J. Appl. Statist., vol. 16, pp. 409-422, 1989.

[6] N. P. Judish, "Polygonal random fields and the reconstruction of piecewise continuous functions," LIDS Rep. LIDS-TH-2176, Laboratory for Information and Decision Systems, MIT, Cambridge, MA, 1993.

[7] S. R. Kulkarni, S. K. Mitter, J. N. Tsitsiklis, and O. Zeitouni, "PAC learning with generalized samples and an application to stochastic geometry." IEEE Trans. Pattern Anal. Machine Intell., vol. 15, pp. $933-942,1993$ 